\title{
The Influence of Trace Elements on the Metabolism of Aromatic Compounds by Soil Fungi
}

\author{
BY MOIRA E. K. HENDERSON \\ The Macaulay Institute for Soil Research, Craigiebuckler, Aberdeen
}

(Received 22 April 1960)

SUMMARY

The effect of copper, iron, manganese and zinc on the metabolism of catechol, $o$-, $m$ - and $p$-hydroxybenzoic, $o$-, $m$ - and $p$-methoxybenzoic, 3:4-dimethoxybenzoic and vanillic acids was investigated. Aspergillus niger, Hormodendrum sp. and Penicillium sp. mycelia which were deficient in the various trace elements were the organisms used. Only iron had any marked effect on the rates of metabolism and on the accumulation of intermediate products of metabolism in the substrate solutions. The intermediate products identified were: protocatechuic acid from $m$ and $p$-hydroxybenzoic and $p$-methoxybenzoic acids; catechol from $o$-hydroxybenzoic acid; vanillic acid from 3:4-dimethoxybenzoic acid; $o-, m$ - and $p$-hydroxybenzoic acids from the corresponding mono-methoxybenzoic acids.

\section{INTRODUCTION}

In the present paper the investigations previously reported (Henderson \& Farmer, 1955; Henderson, 1957) about the metabolism of aromatic compounds by fungi have been continued with a study of the influence of copper, iron, manganese and zinc on their metabolism. By using mycelia deficient in these elements it has been possible to obtain further information on metabolic pathways. The principal test organism was Aspergillus niger which has been so widely used in trace element work. It was established initially that this organism metabolizes aromatic compounds by the same pathway as previously reported for other soil fungi (Henderson \& Farmer, 1955).

\section{METHODS}

Organisms. The following fungi were used: Aspergillus niger M (obtained from Dr E. G. Mulder, Groningen, Holland); Hormodendrum sp. no. 1 from sand under Scots Pine in an afforested sand dune; Penicillium sp. no. 13 from moorland soil. The last two organisms were used in previous investigations (Henderson \& Farmer, 1955; Henderson, 1956, 1957).

Media. The basal growth medium was that of Donald, Passey \& Swaby (1952) from which trace elements were removed by autoclaving with alumina and to which solutions of the required trace elements were subsequently added in the form of Matthey 'Specpure' $\mathrm{H}_{3} \mathrm{BO}_{3}, \mathrm{FeSO}_{4} \cdot 7 \mathrm{H}_{2} \mathrm{O}, \mathrm{CuSO}_{4} .5 \mathrm{H}_{2} \mathrm{O}, \mathrm{ZnSO}_{4} .7 \mathrm{H}_{2} \mathrm{O}, \mathrm{MnSO}_{4} .5 \mathrm{H}_{2} \mathrm{O}$, and $\left(\mathrm{NH}_{4}\right)_{6} \mathrm{Mo}_{7} \mathrm{O}_{24} \cdot 4 \mathrm{H}_{2} \mathrm{O}$ (Johnson, Matthey and Co. Ltd, Hatton Garden, London). This medium was modified for Hormodendrum sp. and Penicillium sp. For these, $0.5 \%(w / v)$ of Difco vitamin-free Casamino acids was added and for the Penicillium 
the medium was adjusted to pH 5. Pure cane sugar was used. Pyrex flasks were used throughout and all glassware was soaked in $\mathrm{HCl}$ solution $(c .17 \cdot 7 \%, \mathrm{w} / \mathrm{v})$ and then rinsed five times with double-distilled water from a glass still. The trace element deficiency of each batch of medium was tested by growing Aspergillus niger on it in static culture and noting the resulting growth.

Inocula. Trace element deficient spores of Aspergillus niger were obtained from 7-day growth on $10 \mathrm{ml}$. liquid medium $\left(\mathrm{NaNO}_{3}, \mathbf{0 . 3} \mathrm{g}\right.$; $\mathrm{KH}_{2} \mathrm{PO}_{4}, 0 \cdot 1 \mathrm{~g}$; $\mathrm{KCl}$, 0.05 g.; $\mathrm{MgSO}_{4} .7 \mathrm{H}_{2} \mathrm{O}, 0.05$ g.; glucose, 1 g.; water, $100 \mathrm{ml}$.), which had been treated with alumina. The growth was transferred to test tubes containing $15 \mathrm{ml}$. 1/1000 $(v / v)$ Tween 80 solution made up with double-distilled water. On shaking the spores became detached from the hyphae and were poured off suspended in the Tween solution. Subsequent preparation of the suspensions was as described by Henderson (1956). The suspensions for static culture were adjusted to contain $3.5 \times 10^{7}$ spores $/ \mathrm{ml}$. and for shaken culture $5 \times 10^{8} / \mathrm{ml}$; $0.1 \mathrm{ml}$. suspension was added to each flask of medium. Sufficient sporing growth of Hormodendrum sp. and Penicillium sp. could not be obtained by the above method and cultures growing on cellophan strips laid on potato glucose agar were used. Spore suspensions of these two organisms were adjusted to contain $6 \times 10^{8}$ spores $/ \mathrm{ml}$.

Cultural conditions. Aspergillus niger was grown by both static and shaken culture. Fungal mats were obtained from static cultures by inoculating $25 \mathrm{ml}$. growth medium, contained in $100 \mathrm{ml}$. conical flasks, and incubating for 6 days at $26^{\circ}$. A thick hyphal mat, densely covered with spores, was obtained in the presence of all trace elements, while in the absence of specific elements the resulting growths were similar to those reported by Donald et al. (1952). Pellets were produced when similar flasks were incubated on a shaking machine (Webley \& Duff, 1955) for 3 days. Hormodendrum sp. and Penicillium sp. were grown only in shaken culture and required incubation periods of 5 and 3 days, respectively.

Replacement technique. After the growth period the culture medium was decanted and the hyphal mats of Aspergillus niger from static culture and the pellets of the three species from shaken culture were washed three times with sterile double-distilled water before adding $25 \mathrm{ml}$. 0.01 M-substrate solution per flask. Solutions of salts of the acids under investigation were prepared at $\mathrm{pH} 6.5$ and treated for removal of trace elements by autoclaving with alumina, and then sterilized by autoclaving. Catechol, which could not be autoclaved, was dissolved in doubledistilled water and sterilized by filtration through a sintered glass filter which had been treated with $\mathrm{HCl}$ for the removal of trace elements. Mats were incubated at $26^{\circ}$ for 1, 2, 3 and 5 days and pellets for 1, 2 and 3 days in the presence of substrate solutions. The mycelium was then filtered off and the solutions acidified and extracted with ether.

Estimation of residual substrates and intermediates. The ether extracts were evaporated to dryness and the residues dissolved in 8 drops of absolute ethanol and equal samples of the ethanolic solutions were applied to Whatman No. 1 filter paper. The papers were developed with $n$-butanol/ammonia (sp.gr. $0 \cdot 880$ )/water (80/5/ $15 \mathrm{ml}$.) during $16 \mathrm{hr}$. at $21^{\circ}$, by the descending method. They were sprayed with diazotized sulphanilic acid (Bray, Thorpe \& White, 1950), ammoniacal silver nitrate or buffer spray (Fewster \& Hall, 1951) for the detection of residual substrates, and of intermediates when these were known from previous investigations (Henderson 
\& Farmer, 1955; Henderson, 1957). When deficiency of a particular metal was affecting metabolism, the differences in rates of metabolism were so distinct that they could readily be detected by examination of the size and intensity of spots obtained on paper chromatograms. Occasionally residual substrates were estimated spectrometrically, as was done for o-hydroxybenzoic acid. Following the extraction of catechol (see later) the substrate solutions were acidified and extracted with ether. The products obtained on evaporation of the ether were dissolved in $0.05 \mathrm{~N}$ $\mathrm{NaOH}$ and the acid was estimated by ultraviolet absorption measurements at $295 \mathrm{~m} \mu\left(\epsilon=3.53 \times 10^{3}\right)$.

Protocatechuic acid, when formed as an intermediate from $m$ - and $p$-hydroxybenzoic acids, was estimated as follows. Products from ether extracts of substrate solutions were dissolved in a known volume of ethanol and $\mathbf{0 . 0 3 3}$ ml. applied to No. 54 Whatman paper by a micropipette. The papers were developed with propanol saturated with borax buffer $(\mathrm{pH} 8$ ) for $7 \mathrm{hr}$., dried, then further developed with butanol saturated with borax buffer $(\mathrm{pH} 8$ ) for $24 \mathrm{hr}$. to obtain good separation of residual hydroxybenzoic acid from protocatechuic acid. The position of protocatechuic acid was located by its fluorescence in ultraviolet radiation and the strip of paper containing the acid cut out. The acid was extracted by immersing the paper in $5 \mathrm{ml}$. borax buffer ( $\mathrm{pH} 8$ ) held at $50^{\circ}$ for $15 \mathrm{~min}$. Protocatechuic acid was estimated by ultraviolet absorption measurements at $293 \mathrm{~m} \mu$, where the acid has an $\epsilon$ maximum of $c .6 .3 \times 10^{3}$.

Catechol was estimated by taking culture solutions to $\mathrm{pH} 7-8$ with $\mathrm{NaHCO}_{3}$, and extracting with ether to remove the catechol. After washing, the ether extracts were taken to dryness and the residues dissolved in borax buffer $(\mathrm{pH} 8)$. Catechol was estimated from ultraviolet absorption measurements at $280 \mathrm{~m} \mu$ where $\epsilon$ is approximately $3.97 \times 10^{3}$.

\section{RESULTS}

Influence of copper, iron, manganese and zinc on the metabolism of o-, m-, and p-hydroxybenzoic, o-, m- and p-methoxybenzoic, 3:4-dimethoxybenzoic and vanillic acids and catechol by Aspergillus niger

Only iron-deficient mats showed a rate of metabolism which was consistently slower on all substrates than was that of the control. Under non-deficient mats the substrates had usually disappeared by 3 days, while under iron-deficient mats substrates were still present after 5 days, frequently in large amounts. This may be partly attributed to the decreased amount of growth in the iron-deficient cultures. However, the growth yields in zinc-deficient cultures were similar to those of irondeficient cultures, yet there was not the consistent markedly decreased rate of metabolism. This points to the participation of iron in the initial stages of metabolism. The amounts of growth were not so markedly decreased in the absence of copper and manganese, although the types of growth were distinct-the pale buff-coloured sporulation of copper-deficient cultures and the 'cauliflower' growth, with almost no sporulation, of manganese-deficient cultures. Copper and manganese did not appear to influence the rates of metabolism.

The experiments with $0-, m$-, and $p$-hydroxybenzoic acids and catechol were repeated with pellets from shaken cultures. Metabolism of pellets was more rapid than was that of mats and the results were not so well defined. However, the same 
trend was obvious: metabolism by iron-deficient pellets was delayed and the disappearance of all four substrates took about twice as long as with non-deficient pellets. This is illustrated in Table 1 by figures obtained for residual $o$-hydroxybenzoic acid when it was being metabolized by non-deficient and iron-deficient pellets. Slight delays sometimes occurred with manganese and zinc deficiencies.

Table 1. Metabolism of o-hydroxybenzoic acid and formation of catechol by shaken cultures of normal and iron-deficient Aspergillus niger

\begin{abstract}
Pellets of the fungus were incubated with $25 \mathrm{ml}$. 0.01 M-o-hydroxybenzoic acid solution at $26^{\circ}$ for the stated number of days. Residual acid and catechol were extracted with ether from substrate solutions and estimated from ultraviolet absorption measurements at 295 and $280 \mathrm{~m} \mu$, respectively.
\end{abstract}

mg. residual $o$-hydroxybenzoic acid/25 ml.

Days
$\mathbf{1}$
$\mathbf{2}$
$\mathbf{3}$

mg. catechol/25 ml.

$\begin{array}{rl}\overbrace{+\mathrm{Fe}} & -\mathrm{Fe} \\ 5.8 & 2.0 \\ <0.02 & 1.8 \\ <0.02 & 1.7\end{array}$

Intermediate products of metabolism were formed by mats and pellets but they were present for a longer period of time and in larger amounts when produced by iron-deficient mycelia than by non-deficient mycelia or by those deficient in copper, manganese or zinc. Also the accumulation of intermediates was less distinct when they were formed by pellets than by mats. The intermediates were: catechol from $o$-hydroxybenzoic acid; protocatechuic acid from $m$ - and $p$-hydroxybenzoic acids; vanillic acid from 3:4-dimethoxybenzoic acid; $o-, m$ - and $p$-hydroxybenzoic acids from the corresponding monomethoxybenzoic acids and, in addition, protocatechuic acid from $p$-methoxybenzoic acid. Catechol was identified by comparison of the infrared spectra of crystalline residues, obtained from ether extracts of substrate solutions, with those of catechol. Figures for the accumulation of catechol formed from $o$-hydroxybenzoic acid by iron-deficient pellets are given in Table 1 . On one occasion protocatechuic acid accumulated during the metabolism of vanillic acid. No intermediate products from catechol were detected by the techniques used. In previous work (Henderson, 1957) the hydroxybenzoic acids, protocatechuic acid and vanillic acid were shown to be intermediates when the corresponding methoxybenzoic acids, $p$-hydroxybenzoic and 3:4-dimethoxybenzoic acids, respectively, were metabolized by Hormodendrum sp. no. 1 and Penicillium sp. no. 13.

Influence of iron, manganese and zinc on the metabolism of o-, $\mathrm{m}-$ and

p-hydroxybenzoic acids and catechol by Hormodendrum sp. and Penicillium sp.

These fungi could not be grown to form mats on the medium used for Aspergillus niger. However, satisfactory growth was obtained by slight modifications of this medium (see Methods) and by incubation on a shaking machine. When the growth medium was replaced by substrate solutions the rate of metabolism of substrates by iron-deficient mycelium of Hormodendrum sp. was approximately half of that by non-deficient mycelium. Zinc and manganese deficiencies did not alter the rate of 
metabolism. Penicillium sp. showed a slightly less-marked delay with iron-deficiency, but it also showed some delay with zinc and manganese deficiencies.

As with Aspergillus niger, intermediate products of metabolism were detected over a longer period of time, and in larger amounts, when formed by iron-deficient mycelia than when formed by non-deficient mycelia or those deficient in manganese or zinc. The accumulation of intermediates was, however, less evident than with A. niger. Paper chromatograms indicated that protocatechuic acid accumulated when it was formed from $m$ - and $p$-hydroxybenzoic acids by the Hormodendrum sp. and Penicillium sp. The amounts present in substrate solutions of Hormodendrum sp. were estimated by ultraviolet spectrometry (Table 2). o-Hydroxybenzoic acid was not metabolized by Hormodendrum sp., while catechol formed from it accumulated in the presence of Penicillium sp. The formation of protocatechuic acid from $m$-hydroxybenzoic acid by Hormodendrum sp. was confirmed by spectrometric examination of extracts from substrate solutions. The position of the absorption maxima at 293 and $253 \mathrm{~m} \mu$ in borate buffer was in agreement with those of a solution of authentic protocatechuic acid.

Table 2. Accumulation of protocatechuic acid, formed as an intermediate product in the metabolism of $\mathrm{m}$ - and $\mathrm{p}$-hydroxybenzoic acids, by shaken cultures of normal and iron-deficient Hormodendrum sp.

Pellets of the fungus were incubated with $25 \mathrm{ml}$. $0.01 \mathrm{M}-m$ - and $p$-hydroxybenzoic acid solutions at $26^{\circ}$ for the stated number of days. Residues from ether extracts of substrate solutions were applied quantitatively to paper chromatograms. After development of the papers, protocatechuic acid was extracted and estimated by ultraviolet absorption measurements at $293 \mathrm{~m} \mu$.

\begin{tabular}{|c|c|c|c|c|}
\hline \multirow[b]{3}{*}{ Days } & \multicolumn{4}{|c|}{$\mu \mathrm{g}$. protocatechuic acid $/ 25 \mathrm{ml}$. } \\
\hline & \multicolumn{2}{|c|}{$+\mathrm{Fe}$} & \multicolumn{2}{|c|}{$-\mathrm{Fe}$} \\
\hline & $\begin{array}{l}m \text {-hydroxy- } \\
\text { benzoic acid }\end{array}$ & $\begin{array}{l}p \text {-hydroxy- } \\
\text { benzoic acid }\end{array}$ & $\begin{array}{l}m \text {-hydroxy- } \\
\text { benzoic acid }\end{array}$ & $\begin{array}{l}p \text {-hydroxy- } \\
\text { benzoic acid }\end{array}$ \\
\hline 2 & 30 & 90 & 60 & 240 \\
\hline 5 & 0 & 0 & 70 & 680 \\
\hline 8 & 0 & 0 & 40 & 420 \\
\hline
\end{tabular}

\section{DISCUSSION}

The principal observation arising from the results is the general requirement for iron in the metabolism of all the aromatic compounds studied. The decreased rate of metabolism of iron-deficient mycelia would lead to a decreased rate of production of intermediate products, yet the intermediates formed in substrate solutions by such mycelia were present over a longer period of time and in larger amounts than were those formed by non-deficient mycelia. This indicates that the rate of metabolism of the intermediates was also decreased. Dagley \& Patel (1957) found that protocatechuic acid oxidase activity could be restored to a dialysed preparation from Pseudomonas sp. by the addition of ferrous ion, but not by several other ions, while Suda, Hashimoto, Matsuoka \& Kamahora (1951) restored activity to a dialysed preparation of pyrocatechase by the addition of ferrous ion. Hayaishi, Katagiri \& Simon (1957) purified pyrocatechase and found that it contained two atoms of 
iron per molecule of enzyme protein. These findings corroborate the present ones, namely, that protocatechuic acid and catechol were more slowly metabolized when formed as intermediate products of metabolism by iron-deficient mycelia than by any others. Copper, manganese and zinc did not seem to play an important role in the metabolism of the aromatic compounds examined.

In addition, evidence has been obtained for the production of protocatechuic acid as an intermediate in the metabolism of $m$-hydroxybenzoic acid, and of catechol as an intermediate of $o$-hydroxybenzoic acid by Aspergillus niger, Hormodendrum sp. and Penicillium sp. Walker \& Evans (1952) reported that Pseudomonas fluorescens oxidized $m$-hydroxybenzoic acid through gentisic acid but Evans (1947) also obtained protocatechuic acid through the action of a Vibrio sp. The former workers also found that $\boldsymbol{P}$. fluorescens grown on $\boldsymbol{o}$-hydroxybenzoic acid was simultaneously adapted to metabolize catechol.

Better defined results were obtained with static mats of Aspergillus niger than with the shaken pellets. It is probable that metabolism is more rapid in the betteraerated shaken flasks and thus the accumulation of intermediates is less likely to occur, while the different rates of metabolism are not so readily distinguished. Also Hormodendrum sp. and Penicillium sp. did not yield as definite results as did A.niger, which may be due either to the fact that trace element deficient spores could not be used or to these species having a lower trace element requirement than $A$. niger. In a study of the growth yields of six fungi pathogenic to tobacco, when various trace elements were omitted, Steinberg (1956) obtained, in the majority of cases, much higher percentage yields than he did with $A$. niger, indicating that the requirements of these fungi were much lower than were those of $A$. niger. The results obtained with Hormodendrum sp. and Penicillium sp. do, however, show that for them also iron is the most important element concerned in the metabolism of the aromatic compounds studied.

I wish to thank Miss D. Brebner for valuable technical assistance and Dr V. C. Farmer and Mr J. D. Russell for carrying out the spectrochemical analyses. I am indebted to Dr E. G. Mulder for the culture of Aspergillus niger and to Dr D. Gross of Tate and Lyle, Ltd. for a gift of pure cane sugar. I also wish to acknowledge the helpful advice of Dr D. M. Webley.

\section{REFERENCES}

Bray, H. G., Thorpe, W. V. \& White, K. (1950). The fate of certain organic acids and amides in the rabbit. 10. The application of paper chromatography to metabolic studies of hydroxybenzoic acids and amides. Biochem. $J .46,271$.

Dagley, S. \& Patel, M. D. (1957). Oxidation of $p$-cresol and related compounds by a Pseudomonas. Biochem. J. 66, 227.

Donald, C., Passey, B. I. \& Swaby, R. J. (1952). A comparison of methods for removing trace metals from microbiological media. J. gen. Microbiol. 7, 211.

Evans, W. C. (1947). Oxidation of phenol and benzoic acid by some soil bacteria. Biochem. J. 41, 373.

Fewster, M. E. \& Hall, D. A. (1951). Application of buffered solvent systems to the detection of aromatic acids by paper partition chromatography. Nature, Lond. $168,78$.

Hayaishi, O., Katagiri, M. \& Simon, R. (1957). Studies on oxygenases: pyrocatechase. J. biol. chem. 229, 905. 
Henderson, M. E. K. (1956). A study of the metabolism of phenolic compounds by soil fungi using spore suspensions. J. gen. Microbiol. 14, 684 .

Henderson, M. E. K. (1957). Metabolism of methoxylated aromatic compounds by soil fungi. J. gen. Microbiol. 16, 686.

Henderson, M. E. K. \& Farmer, V. C. (1955). Utilization by soil fungi of $p$-hydroxybenzaldehyde, ferulic acid, syringaldehyde and vanillin. J. gen. Microbiol. 12, 37.

Steinberg, R. A. (1956). The role of metals in the growth of fungi. Nat.Acad.Sci.Wash., Nat. Res. Counc. Publ. No. 514, 1.

Suda, M., Hashimoto, K., Matsuoka, H. \& Kamahora, T. (1951). Further studies on pyrocatechase. J. Biochem. (Japan), 38, 289.

Walker, N. \& Evans, W. C. (1952). Pathways in the metabolism of mono-hydroxybenzoic acids by soil bacteria. Biochem. J. 52, xxiii.

Webley, D. M. \& DuFF, R. B. (1955). A convenient shaking machine for growing microorganisms. J. appl. Bact. 18, 122. 